\title{
Modeling simulation and virtual platform construction of crossbow making technology
}

\author{
Cong $\mathrm{Wen}^{1}$, Wu Xu ${ }^{1 *}$, Wenquan Tang ${ }^{1}$, Xing Guo ${ }^{1}$, Bing Zhou ${ }^{2}$ \\ ${ }^{1}$ School of Electric and informative Engineering, Yunnan Minzu University, Kunming, Yunnan, 650504, China \\ ${ }^{2}$ Yunnan Agricultural University, Kunming, Yunnan, 650500, China
}

\begin{abstract}
In this paper, a set of virtual simulation platform for crossbow making is established. First, build a virtual model of Lisu crossbow, optimize the model, accelerate the organization, model generation, modification and editing of the database; then complete the database establishment of the corresponding model, nest the comment entity class and filemeta entity class into the file entity class, store them in a nested data set, and complete the data storage, management and query operations; finally, combined with Kalman filtering, arithmetic average filtering and $\mathrm{KNN}$ algorithm are used to build the quest 3D virtual platform to improve the 3D interaction effect with data gloves and complete the construction of the 3D platform.
\end{abstract}

\section{Introduction}

Yunnan is rich in intangible cultural heritage resources. Crossbow is a primitive hunting tool. However, due to the invasion of foreign culture, the technology of crossbow making appears "shrinking", "fault" and even lost [1].

In this paper, we first use Multigen Creator to create the three-dimensional model of crossbow making. Its core is 25 different image generators, which can meet the requirements of database organization, model generation, modification and editing. The main steps of $3 \mathrm{D}$ scene construction include: 1) using camera or scanner to record the crossbow making process, removing useless data, processing data, and determining the scene database hierarchy; 2) importing data into Multigen Creator tool, 3D solid model construction; 3) model integration optimization, testing, etc. Then create the mongodb database. The metadata is stored in the gridfs file system of mongodb by using filemeta entity class. Aiming at the file entity class, the index of Category attribute is established. When querying documents by classification, high efficiency can be achieved. Finally, a digital virtual interactive system based on data glove is established. Kalman filter and arithmetic average filter are used to filter the collected signals and remove noise interference. Finally, k-nearest neighbor algorithm (KNN) is used to recognize the extracted data and improve the final effect of the interactive system.

With the development of computer technology, virtual reality technology is gradually improving information technology. Integrating traditional media methods such as text, image and image into virtual reality display system not only retains the advantages of previous media methods, but also realizes the comprehensive application of various media methods through integration [2].

\section{2 digital protection manual crossbow making technology}

\subsection{Creation of Three-Dimensional Model of "Lisu Crossbow"}

Taking the die used in the process of making Lisu crossbow as an example, the three-dimensional model of the die was established. The modeling tool used is MultiGen Creator. MultiGen Creator is a set of high fidelity and optimal modeling tools. Many important VR development environments are compatible with it. This convenient combination accelerates the organization of database, model generation, modification and editing [3]. The main steps of three-dimensional scene construction include:

1) Pre-preparation: recording the crossbow-making process with camera or scanner, eliminating useless data, processing data, and determining the hierarchical structure of scene database;

2) Modeling: Importing data into MultiGen Creator tool and building three-dimensional entity model;

3) Post-processing: model integration optimization, testing, etc.

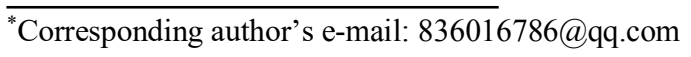




\subsubsection{Data acquisition of "Lisu Making Crossbow" die}

The original data is transmitted to the computer through a digital camera or scanner. It is then converted into a format texture file available to MultiGen Creator. The texture data used in the scene model of this system mainly come from the pictures in the field photographs and material picture library.

\subsubsection{Data processing}

After the completion of data collection, the collected data need to be processed preliminarily: remove some incorrect and unnecessary data, then transform and cut the data, finally integrate the data into a database according to a certain format, and classify and store the texture data for reserve.

\subsubsection{Scenario database hierarchy}

Firstly, the hierarchical structure of all entity models in the virtual scene is determined according to the structural relationship between the models and the interior of the model. Then, the general structure of the virtual scene is determined by data acquisition. According to the distribution of sampled features, the whole scene area is segmented.

\subsubsection{Construction of Static Model}

Static entity modeling mainly uses geometric modeling technology, using modeling tools to simulate the entity from shape and appearance, and using a large number of texture mapping and other auxiliary technical means to reduce the complexity of the model. Then each important module is modeled separately. The virtual model of Lisu nationality's crossbow making is obtained as shown in Fig. 1.

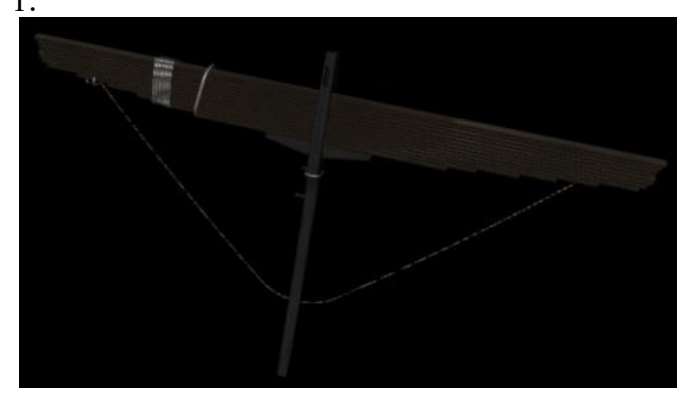

Fig. 1 Static model of Lisu crossbow

\subsubsection{Construction of Dynamic Model}

For the grinding steps of wood and the connection between different modules in crossbow making process, motion modeling is needed. The DOF nodes of the moving parts should be added to the files formed by the modeling, and the motion relations of multiple moving parts should be carried out on the basis of DOF, so as to determine the motion traction relations.

\subsection{Create MongoDB database}

\subsubsection{Fixed set data type}

Several entity classes have been designed in the design of key data model. Using the characteristics of nested data model in MongoDB database system, Comment entity class and FileMeta entity class can be nested in File entity class and stored in a nested data set. MongoDB database system only needs two sets to complete the data storage model of the core business module of the system.

\subsubsection{Data storage and management}

The metadata of document data is stored in the GridFS file system of MongoDB using FileMeta entity class. GridFS file system uses binary mechanism to store document data in knowledge sharing platform. The original file data of documents in knowledge sharing platform and the SWF file data that can be read online by WEB browser client after conversion are stored by GridFS file system. The original data and SWF file data can be read and written by creating and writeTo methods in the API interface of MongoDB [4].

The GridFS file system also provides data structure support in the form of collections, which can perform corresponding operations like common collections. GridFS file system can divide the whole large file binary data into smaller blocks, each of which is treated as a separate document for storage.

\subsubsection{MongoDB database query operation}

For File entity classes, we can build an index for Category attributes, which can achieve high efficiency when querying documents according to classification. Knowledge sharing system can establish multiple indexes according to common query conditions, which can greatly improve the performance of multi-condition query.

\subsection{Digital Gesture Operation System Based on Data Glove}

In order to enhance the interaction between users and virtual objects, a digital virtual interactive system based on data gloves is designed. Users can use this system to experience all the processes of crossbow control. The data glove model used in this system is 5DT Data Glave 14 Ultra. Fig. 2 is a physical picture of data gloves. 


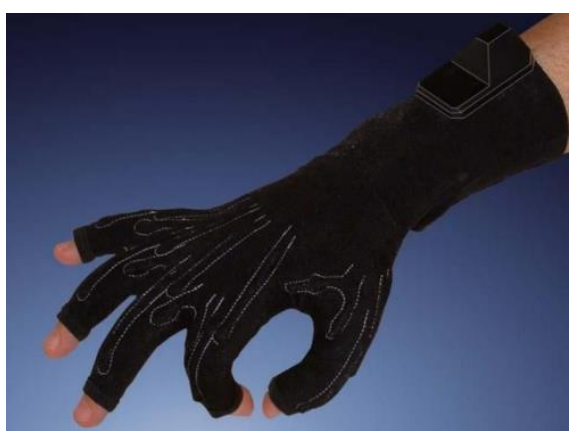

Fig. 2 Data Gloves

The data of data gloves can be acquired by hardware system design, but because the data acquisition of data gloves is more complex, the first step is to filter the collected signals to remove noise interference. Finally, gesture recognition of the extracted data is carried out by K-nearest algorithm (KNN) [5].

\subsubsection{Kalman filter}

Kalman filter estimates the state variables by solving the state space model of signal and noise, using the estimated values and the current observations, and then gets the current estimates. Kalman filter can reduce the data deviation collected, improve the piercing phenomenon in virtual environment, and improve the accuracy of collision feedback.

\subsection{2 arithmetic average filtering method}

In the design of the system, acceleration data and angular velocity data collected need to be transferred to external storage units for identification. Therefore, the stability and integrity of data transmission will affect the accuracy of identification. The scheme adopts wireless transmission mode, and the sampling frequency of accelerometer and gyroscope are $100 \mathrm{~Hz}$. Wireless transmission is easily disturbed by environmental noise. The arithmetic average filtering method can remove noise and improve signal-to-noise ratio.

\subsubsection{KNN algorithm}

KNN algorithm classifies the samples according to the distance relationship between the eigenvalues. If the nearest sample in the feature space of a sample data belongs to a certain category, then the sample belongs to the same category. By classifying the eigenvalues and selecting the required eigenvalues as the reference data for gesture recognition, the virtual model can be accurately controlled.

\subsection{Quest3D Digital Virtual Reality Platform Construction}

All editors in Quest3D are visual. It encapsulates a lot of visual modules, and the programming interface is highly visualized. It does not need to write thousands of lines of complex code. It can effectively complete the development of virtual reality system and reduce the use threshold[6].

Firstly, we use MultiGen Creator software to model Lisu's crossbow-making. The texture maps corrected in Photoshop are endowed with 3D models, the correct UV is set, and the scenes are integrated after lighting and baking maps are arranged in the 3D sMax. After scene integration, the three-dimensional model is converted into. X format supported by Quest3D through plug-in, and then imported into Quest3D editor smoothly. Then, the system functions are realized through component module development, such as scene roaming, human-computer interaction and special effect editing[7].

After the completion of the platform, after continuous testing and optimization, QuestViewer can be generated to play *. Q3D files or released into independent executable $*$. exe files.

\section{Conclusion}

In this paper, a virtual reality interactive platform for Lisu handmade crossbows is developed by using virtual reality technology. The virtual interactive system allows the experiencer to watch the whole process of crossbow control and imitate the representation of the virtual crossbow to realize the purpose of learning and inheriting the crossbow control technology. It is of great significance for the protection of national spiritual wealth to seek the conjunction point between modern science and traditional handicraft. It is expected that virtual reality technology will become an important way to protect traditional handicraft and provide more effective and feasible ways to protect traditional handicraft.

\section{Acknowledgments}

This paper is supported by the National Natural Science Joint Fund project "Computational Support Technology and Empirical Study of Yunnan Frontier Ethnic Culture in the Big Data Environment" (U1802271) and the National Nationalities Commission Ethnic Research Foundation project "Research on Diversified Protection and Development of Ethnic Minority Culture in the Background of Big Data" (2019-GMD-056).

\section{References}

1. Tian Shichang, Wang Mingye, Mu Shunbi, Zhang Aihua. Investigation and analysis of the status quo of traditional sports cultural identity of Lisu nationality in Yunnan [J].Sports World (Academic Edition), 2019 (03): 64+90.

2. Xiangqian, Guo Liyao, Li Jiangbo. Research on the protection strategy of intangible cultural heritage of ethnic minorities based on virtual reality technology --- Take the Miao people's Autumn Festival as an example [J]. Computer knowledge and technology, 2016, 12 (25): 210-213. 
3. Zhai Liping. Research and implementation of virtual reality three-dimensional modeling technology based on MultiGen [D]. Chongqing University, 2005.

4. Gao Liqun, Yang Bo. Application of virtual reality technology in intangible cultural heritage protection in Hebei Province [J]. Grand Stage, 2015 (03): 231-232.

5. Gong Wen, $\mathrm{Xu}$ Junjun, Yang Juekuan. Force feedback data glove system and master-slave control research $[\mathrm{J}]$. Mechanical design and manufacturing engineering, 2019, 48 (06): 56-59.

6. Yang Xiaoqin. Practice and thinking of virtual reality technology based on Quest3D platform [J]. China Education Informatization, 2017 (14): 87-91.

7. Problems and Countermeasures in the digitalization construction of Yuan Yanlei-Lisu archives [J]. Lantai World, 2019 (06): 28-30. 